\title{
Desenvolvimento e validação preliminar da Escala Situacional de Satisfação Corporal - ESSC
}

\author{
Elizabeth Hirata ${ }^{1}$ - Universidade de Brasília, Brasilia, Brasil \\ Ronaldo Pilati - Universidade de Brasilia, Brasília, Brasil
}

\begin{abstract}
Resumo
O objetivo deste estudo foi desenvolver e validar um instrumento de satisfação corporal situacional numa amostra de estudantes universitários brasileiros. A escala inclui itens de avaliação de partes e do corpo como um todo. Análises fatoriais exploratórias revelaram uma estrutura com quatro fatores; "partes inferiores" $(\alpha=0,72)$, "satisfação e músculo" $(\alpha=0,82)$, "partes externas" $(\alpha=0,65)$ e "insatisfação e gordura" $(\alpha=0,82)$. Seus 28 itens obtiveram cargas fatoriais variando entre 0,32 e 0,82 . Análises adicionais indicaram que a escala foi sensível a características da amostra, como Índice de Massa Corporal e gênero, corroborando a literatura atual da área. Palavras-chave: Imagem corporal, Escala de imagem corporal, Satisfação corporal, Insatisfação corporal, Gênero.
\end{abstract}

\section{Development and preliminary validation of the Body Satisfaction Situational Scale - ESSC}

\begin{abstract}
The aim of this study was the development and validation of a situational body satisfaction scale in a Brazilian college student sample. The scale includes 28 items ranging from parts to overall body evaluation. Four factors emerged from the exploratory factor analysis of the items. They were named "lower parts" $(\alpha=.72)$, "muscle and satisfaction", $(\alpha=.82)$, "external parts" $(\alpha=.65)$ and "fat and dissatisfaction" $(\alpha=.82)$. The factor loadings of its 28 items ranged from .32 to .82. Further analysis has indicated the scale was sensitive to the sample's characteristics, such as, Body Mass Index and gender, according to the current field's literature.

Keywords: Body image, Body image scale, Body satisfaction, Body dissatisfaction, Gender.
\end{abstract}

A importância subjetiva dada ao corpo pode estar motivando um fenômeno que se observa no país. Atualmente, a venda de remédios para emagrecer (anorexígenos) e as taxas de cirurgias estéticas estão entre os primeiros lugares do mundo. Por trás desses altos índices estatísticos, encontra-se o comportamento de milhares de indivíduos. Por algum motivo, eles desejam perder peso e modificar seus corpos, levando a esse quadro alarmante.

Nesse contexto, a imagem corporal assume um papel central. Pruzinsky e Cash (2002) defendem que o aspecto fundamental da imagem corporal reside na sua capacidade de influenciar substancialmente a qualidade de vida. Talvez, mais marcadamente, ela influencie nossos relacionamentos. Algumas abordagens teóricas têm estudado a imagem corporal, como a psicodinâmica, a neurocognitiva e a feminista (Pruzinsky \& Cash, 2002). Dentre elas, a sociocultural defende a influência de aspectos culturais na percepção e autoavaliação do corpo.

\footnotetext{
1 Agradecemos ao grupo de psicometria do professor Jacob Arie Laros, incluindo Fabiana Queiroga, Josely Gomes, Josemberg Moura de Andrade e Juliana Siqueira; ao professor Jairo E. BorgesAndrade e aos alunos da disciplina de elaboração de artigos científicos de janeiro de 2009 do PPG/PSTO.

Endereço para correspondência:

SQN 104 bloco G apto. 507 - 70733-70 - Brasília-DF

E-mail: bethhir@gmail.com
}

Essa abordagem foca, principalmente, no impacto da norma social de magreza feminina e, recentemente, do ideal masculino de musculosidade, na satisfação corporal (Cafri, Yamamiya, Brannick \& Thompson, 2005; Grogan, 2008; Jackson, 2002; Thompson, Van den Berg, Roehrig, Guarda \& Heinberg, 2004). Com base nela, muitos estudos experimentais observaram efeitos negativos da exposição às imagens de corpos idealizados na satisfação corporal (Groesz, Levine \& Murnen, 2002).

Diversas pesquisas têm encontrado relações entre (in)satisfação corporal e muitas variáveis. É o caso do comportamento alimentar (Pelletier \& Dion, 2007), tendência a transtornos alimentares (Kim \& Lennon, 2007; Pelletier \& Dion, 2007; Stice, 1994), depressão (Keel, Mitchell, Davis \& Crow, 2001), autoestima (Van den Berg \& colaboradores, 2007), comparação social (Strahan, Wilson, Cressman \& Buote, 2006; Van den Berg \& colaboradores, 2007) ansiedade frente à aparência social (Hart \& colaboradores, 2008) e índice de massa corporal (Durkin, Paxton \& Sorbello, 2007).

O entendimento da satisfação individual com o próprio corpo é digno de consideração. Traz informações de um fenômeno cujos impactos se observam no nível de análise coletivo e no individual. Contudo, o seu estudo no país requer o 
uso de medidas brasileiras confiáveis e apropriadas. Como a satisfação corporal tem influências culturais, o seu entendimento requer a utilização de medidas adaptadas ao contexto local. Há uma escassez de questionários e escalas em português. O mesmo não se observa na literatura internacional, que oferece grande diversidade dessas medidas (Grogan, 2008). A multiplicidade desses instrumentos, criados ao longo do tempo, permitiu que algumas das suas falhas fossem apontadas e servissem de diretrizes na elaboração de novas medidas.

O presente estudo inicia abordando as definições conceituais de imagem e satisfação corporal. Segue-se uma breve revisão de literatura sobre os instrumentos de medida de imagem corporal. Por fim, apresenta-se a medida aqui proposta.

\section{Imagem corporal e satisfação corporal}

Entende-se satisfação corporal a partir da esfera conceitual na qual ela se insere, a imagem corporal. Atualmente, muitos estudiosos da imagem corporal concordam que ela é um construto multidimensional (Pruzinsky \& Cash, 2002; Thompson, 2004), que abrange aspectos cognitivos, atitudinais e comportamentais (Grogan, 2008; Pruzinsky \& Cash, 2002). Definese aqui satisfação corporal como um aspecto atitudinal da imagem corporal. Trata-se das avaliações cognitivas e afetivas de uma pessoa sobre seu corpo inteiro e suas partes, em função das suas diversas características, como tamanho, forma, peso, massa muscular, dentre outros (Grogan, 2008). Uma vez que atitudes são avaliações de cunho favorável ou desfavorável sobre algo (Eagly \& Chaicken, 1998), assume-se que a satisfação seja um posicionamento positivo ante o corpo. $O$ oposto ocorre na insatisfação corporal. Nesse sentido, ela assume polaridades. Muitos autores estudam e/ou definem diretamente a avaliação negativa do corpo com o termo "insatisfação corporal" (Cash, Fleming, Alindogan, Steadman \& Whitehead, 2002; Grogan, 2008; Pelletier \& Dion, 2007; Scagliusi \& colaboradores, 2006; Sepúlveda, Botella \& León, 2002; Strahan, Wilson, Cressman \& Buote, 2006; Trampe, Siero \& Stapel, 2007; Van den Berg \& colaboradores, 2007). Segundo Grogan (2008), insatisfação corporal seria caracterizada por "pensamentos e sentimentos negativos de uma pessoa sobre seu corpo".

Instrumentos de medida de satisfação corporal
De modo geral, a satisfação corporal tem sido medida com base em instrumentos que avaliam a percepção visual ou por meio de escalas atitudinais. Dentre os primeiros, podem ser citadas as escalas de figuras de silhuetas (Fingeret, Gleaves \& Pearson, 2004; Kakeshita \& Almeida, 2006; Scagliusi \& colaboradores, 2006). Essas apresentam figuras de corpos dispostos em ordem crescente de gordura ou massa muscular. A diferença entre as silhuetas indicadas como o corpo atual e como corpo ideal tem sido entendida como medida de insatisfação corporal. Usando esse raciocínio, recentemente foram criados programas de computador. Eles permitem maior precisão na estimativa das medidas corporais (Harari, Furst, Kiryati, Caspi \& Davidson, 2001; Kagawa, Kerr, Dhaliwal, Hills \& Binns, 2006).

Em relação às medidas atitudinais, Thompson (2004) argumenta que a elaboração de um instrumento de imagem corporal deve ser bastante específica no tocante à dimensão do construto tratado. Alguns instrumentos avaliam a satisfação ou com partes específicas com o corpo todo. Segundo o autor, ao se generalizarem os dados obtidos num instrumento que utiliza apenas um nível como se fosse satisfação geral, podem-se produzir conclusões errôneas. Medindo partes do corpo, tem-se o Eating Disorder Inventory - EDI, que possui um fator de "insatisfação corporal". Seus itens são sobre partes do corpo, como "penso que as minhas ancas são muito largas". Proposto por Garner, Olmstead e Polivy (1983), ele foi validado em amostras femininas, seu alfa foi de 0,90 em pacientes com anorexia e de 0,91 num grupo controle. Ele foi traduzido e adaptado para o português (Costa, Ramos, Barros, Torres, Severo \& Lopes, 2007). O Multidimensional BodySelf Relations Questionnaire possui uma subescala, a The Body Areas Satisfaction Scale - BASS (Cash, 2000). As respostas dessa escala de 5 pontos variam entre "muito insatisfeito(a)" a "muito satisfeito(a)". Os itens avaliam partes do corpo e alguns aspectos, como tônus muscular. Possui um alfa de 0,77 para homens e 0,73 para mulheres. Contudo, não possui versão em português e não pode ser modificada ou alterada, por direitos autorais. A escala unidimensional Body Appreciation Scale - BAS (Avalos, Tylka \& Wood-Barcalow, 2005) apresenta alfas de 0,91 e 0,93 em dois estudos. A Escala de Avaliação da Satisfação com a Imagem Corporal - EASIC (Ferreira \& Leite, 2002) é um instrumento nacional bifatorial. Bem como a BAS, a EASIC mede apenas a apreciação geral do corpo. Possui um fator "satisfação com a 
aparência" e a "preocupação com o peso" com alfas de 0,90 e 0,79, respectivamente. Entretanto, a escala é adequada apenas para mulheres, com itens como "Estou sempre preocupada com o fato de poder estar gorda".

Ainda não foram encontradas na literatura medidas que façam sentido em amostras compostas por homens e mulheres. Muitos instrumentos são mais apropriados à satisfação corporal feminina. É o caso da EASIC, do Body Shape Questionnaire - BSQ (Cooper, Taylor, Cooper \& Fairburn, 1987) e do fator de insatisfação da EDI. O BSQ foi desenvolvido com base em entrevistas com mulheres, incluindo pacientes com transtornos alimentares. Ele possui uma versão em português, não validada (Freitas, Gorestein \& Appolinário, 2002). Embora seja uma medida de preocupações corporais, em especial com a experiência de se "sentir gorda" (Cooper \& cols., 1987), o BSQ tem sido entendido como medida de satisfação corporal (Freitas \& cols., 2002). Porém, um instrumento que avalie especificamente a preocupação com a gordura não parece adequado para medir satisfação corporal em homens e mulheres. Eles valorizam diferentes aspectos e partes do corpo (Standford \& McCabe, 2002; Tylka, Bergeron \& Schwartz, 2005). Diante da escassez de medidas voltadas ao público masculino, foi criada a Male Body Attitudes Scale MBAS (Tylka \& cols., 2005). Feita para medir as atitudes masculinas quanto à aparência corporal, ela possui itens adequados às especificidades da satisfação corporal dos homens. Dois estudos revelaram sua estrutura trifatorial, "musculosidade" ( $\alpha=0,90$ e 0,91), "baixa gordura corporal" ( $\alpha=0,93$ e 0,95$)$ e "altura" $(\alpha=0,81$ e $0,80)$. O alfa total da escala foi de 0,91 . Não foram encontrados instrumentos brasileiros validados avaliando atitudes masculinas em relação ao corpo.

Thompson (2004) sugere o uso de escalas que meçam o estado de satisfação e não o traço de personalidade. Esse cuidado deve ser tomado principalmente em pesquisas experimentais, que buscam medir o efeito de algum estímulo na satisfação corporal. Embora a imagem corporal responda ao contexto, a maioria dos pesquisadores tem tratado a imagem corporal como um traço estável (Cash \& colaboradores, 2002). A Body Image States Scale - BISS (Cash \& colaboradores, 2002) avalia a satisfação corporal momentânea. Contudo, ela não aborda a satisfação com partes específicas do corpo, apenas a satisfação geral. Não foram encontradas medidas validadas em língua portuguesa avaliando a satisfação momentânea.
Embora haja vários instrumentos de medida de diferentes aspectos da imagem corporal, poucos foram encontrados em língua portuguesa. Faltam no contexto nacional escalas que: 1) meçam o estado de satisfação corporal; 2) incluam itens de satisfação com partes do corpo ou do seu todo; 3) tragam itens relevantes tanto à satisfação corporal feminina quanto à masculina. Assim, o objetivo da presente pesquisa foi o desenvolvimento e a validação preliminar de um instrumento do estado de satisfação corporal, a Escala Situacional de Satisfação Corporal - ESSC. Buscou-se aferir a validade de construto da escala desenvolvida. Trata-se de um instrumento novo, que se baseou na adaptação de alguns itens de escalas existentes e na proposição de novos itens. Ela pretende medir a satisfação corporal momentânea em universitários brasileiros de ambos os sexos, com itens sobre partes do corpo e do corpo como um todo.

\section{Método}

\section{Participantes}

A amostra de conveniência escolhida teve dois critérios prévios. O principal baseou-se no projeto de estudo experimental com universitários que utilizou essa escala como medida de variável dependente. Assim, a escala foi validada para a mesma população que fez parte do experimento. Ademais, a amostra permite a investigação do construto com um público não necessariamente portador de distúrbios relativos a questões corporais.

O presente estudo contou com a participação de 451 estudantes universitários matriculados em diversos cursos do Distrito Federal. Ao todo foram 200 homens $(44,3 \%)$ e 251 mulheres. A idade média dos participantes foi de 24 anos $(\mathrm{DP}=6,7)$. A maioria dos estudantes era proveniente de uma universidade pública e 35,3\% de instituições privadas.

\section{Instrumento}

A fim de adaptar os conteúdos dos itens às particularidades da língua portuguesa, não foi utilizada a técnica de tradução-retradução. As escalas originais foram traduzidas em grupo de pesquisa. O grupo, dirigido por um docente, foi composto por três pesquisadores de pósgraduação e dois de graduação. Os membros eram de áreas diversas, incluindo estatística, psicometria, psicologia organizacional e psicologia social. Após uma série de validações semânticas, o 
questionário foi reduzido de 33 para 28 itens, passando, então, por uma validação com juízes. Todo esse processo resultou na inclusão e modificação de diversos itens, como será descrito a seguir.

Segundo Pasquali (1999), a literatura de medidas de um construto é uma possível fonte de definições e de itens. Com base na literatura de medidas de imagem corporal, a satisfação corporal foi definida operacionalmente como opiniões e/ou sentimentos - positivos ou negativos - de um indivíduo sobre seu corpo como um todo, ou a partes dele. Como a maioria das escalas encontradas trazia apenas itens ou de parte ou de corpo inteiro, esses dois aspectos foram entendidos como as dimensões principais. Com base nelas foram adaptadas e elaboradas questões que incluíram avaliações positivas e negativas e características corporais voltadas para ambos os sexos, como atratividade, forma, tamanho, massa muscular e gordura. Após as análises semânticas houve muita mudança na redação dos itens e poucos se mantiveram fiéis às escalas originais utilizadas.

Conforme mencionado anteriormente, alguns itens foram adaptados de escalas existentes. Uma delas foi a escala BAS. Em relação à satisfação geral com o corpo, o item da BAS "Em geral estou satisfeito(a) com meu corpo" foi modificado, retirando-se o "em geral". Dois itens foram adaptados da EASIC, sendo o termo "aparência" substituído por um mais específico, "corpo", como em "Sinto vergonha do meu corpo". Ainda dessa escala, adaptou-se o item "Estou satisfeita com meu peso" para o "Gosto do peso que tenho agora". Sobre a satisfação com partes do corpo, foram utilizados itens do fator de "insatisfação corporal" da versão portuguesa da EDI (Costa \& colaboradores, 2007), adaptando-os ao português do Brasil. Em "Acho que meu estômago é grande demais", substituiu-se a palavra estômago por barriga. Processo semelhante ocorreu com os itens sobre partes inferiores, como quadril e glúteos, e pernas. O item "Estou satisfeito(a) com o tamanho dos meus quadris" foi adaptado da EDI, substituindo-se a palavra "ancas" do instrumento original. A satisfação corporal masculina tomou por base alguns itens da MBAS. Mantiveram-se os itens "Acho que tenho gordura demais no corpo" e "Estou satisfeito(a) com a minha altura". Outros itens foram elaborados, baseados em características e/ou partes do corpo constantes nessa escala, como musculosidade, ombros e costas. Desse modo, a maioria dos itens, 24, dos 28 itens iniciais dessa escala, foi adaptada de outra escala. Outros 5 itens foram propostos em grupo e durante o processo das validações semânticas. Foi o caso dos itens "Acho que tenho celulites demais", "Estou satisfeito(a) com a quantidade de pelos que tenho no corpo" e outros dois envolvendo cabelos e rosto.

A instrução do presente instrumento seguiu a BISS. Ela orienta o participante a responder de acordo com a sua opinião "nesse momento", numa escala Likert, de concordância, de 5 pontos. Ademais, foram elaboradas algumas questões demográficas ao fim do questionário, envolvendo autorrelato de altura e peso (para cálculo do índice de massa corporal, IMC), idade, sexo, curso e universidade.

\section{Procedimentos de coleta e análise dos dados}

A coleta de dados foi feita após a aprovação pelo comitê de ética do instituto de saúde de uma instituição de ensino superior no DF. Ela ocorreu em diversas instituições em horários distintos. Após consentimento dos professores, os alunos foram convidados a participar da pesquisa em sala de aula. Aqueles que concordaram assinaram o termo de consentimento e responderam aos questionários, o que durou, em média, 20 minutos.

Realizaram-se análises descritivas e inferenciais. Foi feita a análise de componentes principais e análise fatorial pelo método de fatoração dos eixos principais. O índice de massa corporal (IMC) foi calculado dividindo-se o peso pelo quadrado da altura.

\section{Resultados}

O presente estudo objetivou desenvolver e posteriormente aferir a validade de construto da escala ESSC. Para tanto, foram realizadas as análises preliminares. A quantidade de outliers multivariados foi pequena $(0,1 \%)$ e decidiu-se pela não-exclusão dos mesmos. A única característica comum entre eles foi que, embora mais da metade da amostra fosse de estudantes de uma instituição pública, mais de $50 \%$ dos outliers multivariados veio de faculdade particular. A média do IMC masculino foi de $24(\mathrm{DP}=3,7)$ e do feminino, de $22(\mathrm{DP}=3,7)$, enquadrando-os na faixa da normalidade ou eutrofia.

$O$ índice de adequação da amostra de Kaiser-Meyer-Olkin (KMO) indicou boa fatorabilidade do instrumento $(\mathrm{KMO}=0,893)$. A 
estimativa inicial do número de fatores foi feita com uma análise de componentes principais. Pôde-se observar no gráfico de sedimentação uma estrutura variando entre 2 e 5 fatores. Foi feita uma análise paralela que apontou 4 fatores, por meio do software RanEigen (Enzmann, 1997).

Conforme Costello e Osborne (2005), em ciências sociais, o uso de rotações oblíquas deve levar a soluções mais acuradas teoricamente, pois assume que o comportamento não é isolado. Partindo-se do entendimento de correlações entre os fatores, foram feitas análises fatoriais com a rotação oblimin e extração PAF (Principal Axis Factoring). Selecionaram-se apenas os itens que obtiveram cargas fatoriais iguais ou acima de 0,32 , seguindo as instruções de Tabachnick e Fidel (1996). Esse parâmetro resultou na exclusão de 5 itens. Dentre eles estavam o "Estou satisfeito(a) com as minhas costas" e "Se eu estivesse na praia, me sentiria bem em roupas de banho".

Após uma investigação indo de 4 a 2 fatores, optou-se pela estrutura composta por 4 fatores. Essa solução, além de ter sido apontada pela análise paralela, também gerou fatores que se agruparam de modo mais coerente no conteúdo. De modo geral, os itens se agruparam tanto em
Hirata, E., Pilati R. Escala Situacional de Satisfação Corporal

5

termos da satisfação e insatisfação quanto em relação a determinadas partes do corpo (ver Tabela 1). A variância total explicada dos 4 fatores foi de 54\%. Sete itens compuseram o primeiro fator. Ele obteve bons índices de confiabilidade interna, com o mais alto alfa de Cronbach da escala. Seu conteúdo pôde ser agrupado em função da carga negativa dos itens, bem como de conteúdos relacionados à gordura. Assim, esse fator foi intitulado de "insatisfação e gordura". O segundo fator, composto por 4 itens, teve o menor alfa da escala. Como seus itens abordam partes específicas do corpo, como cabelos, rosto e pelos, o fator foi denominado "partes externas". Oito itens compuseram o terceiro fator, cujo alfa empatou com o do primeiro fator. Por conter questões de avaliação positiva e também sobre músculos, o fator foi denominado de "satisfação e músculos". Nesse fator, o item de maior carga fatorial foi o "De modo geral, estou satisfeito(a) com o tamanho dos meus músculos". Finalmente, o último fator, com apenas 4 itens, apresentou um alfa maior que o fator 2 e foi denominado "partes inferiores". O conteúdo dos itens, bem como suas cargas fatoriais e comunalidades, podem ser observados na Tabela 1.

Tabela 1 - Cargas fatoriais e alfas de Cronbach da Escala Situacional de Satisfação Corporal - ESSC (continua)

\begin{tabular}{|c|c|c|c|}
\hline Item $\mathrm{n}^{\circ}$ & Item & Cargas fatoriais & Comunalidades \\
\hline \multicolumn{4}{|c|}{ Fator 1: "insatisfação e gordura" $(\alpha=0,82)$} \\
\hline 2 & Gostaria de mudar muitas coisas no meu corpo & $-0,42$ & 0,38 \\
\hline 4 & Sinto vergonha do meu corpo & $-0,35$ & 0,35 \\
\hline 7 & Estou descontente com a minha cintura & $-0,49$ & 0,30 \\
\hline 13 & Se eu estivesse mais magro(a), me sentiria muito melhor & $-0,69$ & 0,55 \\
\hline 18 & Estou descontente com a minha barriga & $-0,71$ & 0,43 \\
\hline 20 & Acho que tenho gordura demais no meu corpo & $-0,82$ & 0,71 \\
\hline 23 & Estou descontente com as minhas medidas & $-0,54$ & 0,55 \\
\hline
\end{tabular}

Fator 2: "partes externas" $(\alpha=0,65)$

21 Estou satisfeito(a) com os meus cabelos corpo

Fator 3: "satisfação e músculo" $(\alpha=0,82)$

3 Estou satisfeito(a) com a grossura dos meus braços 
Tabela 1 - Cargas fatoriais e alfas de Cronbach da Escala Situacional de Satisfação Corporal - ESSC (conclusão)

\begin{tabular}{llcc}
\hline Item $\mathrm{n}^{\circ}$ & \multicolumn{1}{c}{ Item } & Cargas fatoriais & Comunalidades \\
\hline 11 & Gosto da largura dos meus ombros & 0,39 & 0,22 \\
14 & Estou satisfeito(a) com o meu peito & 0,39 & 0,28 \\
15 & Acho meu corpo atraente & 0,44 & 0,46 \\
22 & De modo geral, estou satisfeito(a) com o tamanho dos & 0,72 & 0,61 \\
& meus músculos & & 0,75
\end{tabular}

Fator 4: "partes inferiores" $(\alpha=0,72)$

$5 \quad$ Estou satisfeito(a) com o tamanho dos meus quadris $\quad 0,60 \quad 0,45$

$9 \quad$ Estou satisfeito(a) com os meus glúteos $\quad 0,56 \quad 0,43$

16 Acho que minhas pernas são muito flácidas $\quad-0,48 \quad 0,64$

26 Acho que tenho celulites em excesso $\quad-0,67 \quad 0,43$

A correlação das médias das respostas nos fatores foi significativa entre os quatro. A correlação de maior magnitude foi negativa e envolveu os fatores "insatisfação e gordura" e "satisfação e músculo" ( $r=-0,54, p<0,001)$. O fator "insatisfação e gordura" teve outra correlação negativa média, com o fator "partes inferiores" $(r=-0,52, \quad p<0,001)$. A mais baixa das suas correlações foi com o fator "partes exteriores" $(r=-0,13, \quad p<0,001)$. Esse último obteve as correlações mais fracas com os outros, de modo geral, sendo todas significativas $(p<0,001)$ e abaixo de 0,30 . Os fatores "satisfação e músculo" e "partes inferiores" obtiveram uma correlação média positiva e significativa $(r=-0,45, p<0,001)$.

Pasquali (1999) sugere que algumas maneiras comuns de discriminar itens são os coeficientes de correlação e o teste-t. Foram feitas ambas as análises, indicando que a estrutura se mostrou sensível a variações demográficas relevantes da amostra. Para tanto, foram criados escores fatoriais para cada um dos quatro fatores e relacionados com dados sociodemográficos.
Análises de correlação indicaram relações significativas entre o IMC e o fator "insatisfação e gordura" $(r=0,505 ; p<0,001)$. A segunda maior correlação foi negativa, envolvendo a massa corporal e o fator "satisfação e músculo" ( $r=$ $0,411 ; p<0,001)$. A correlação entre "partes externas" e o IMC foi menor $(r=0,126 ; p=0,51)$. Por fim, "partes inferiores" também teve correlação com o IMC ( $r=-0,275 ; p<0,001)$.

Testes-t revelaram que as médias dos fatores variaram em razão do gênero. No fator "insatisfação e gordura", a média das mulheres $(2,87)$ foi significativamente maior que a dos homens (2,49). No fator "partes externas", os homens tiveram uma média $(3,78)$ significativamente maior que as mulheres $(3,51)$. A diferença de gênero não foi significativa em "satisfação e músculos". Já no "partes inferiores", elas tiveram médias significativamente menores $(3,27)$ do que eles $(4,26)$. Os resultados dessa análise podem ser observados com mais detalhes na Tabela 2.

Tabela 2 - Testes-t para homens e mulheres nos fatores da Escala Situacional de Satisfação Corporal

\begin{tabular}{clcccccc}
\hline \multicolumn{1}{c}{ Nome do fator } & Sexo & $\mathrm{N}$ & Média & Desvio padrão & Erro padrão & $\mathrm{t}$ & Sig. (bicaudal) \\
\hline \multirow{4}{*}{ Insatisfação e gordura } & Homens & 200 & 2,48 & 0,903 & 0,064 & $-4,29$ & $<0,001$ \\
& Mulheres & 251 & 2,87 & 1,000 & 0,063 & $-4,34$ & $<0,001$ \\
\multirow{2}{*}{ Partes externas } & Homens & 200 & 3,78 & 0,843 & 0,060 & 3,37 & 0,001 \\
\multirow{2}{*}{ Satisfação e músculos } & Mulheres & 251 & 3,50 & 0,866 & 0,055 & 3,38 & 0,001 \\
\multirow{2}{*}{ Partes inferiores } & Homens & 200 & 3,39 & 0,816 & 0,058 & 0,62 & 0,533 \\
& Mulheres & 251 & 3,34 & 0,819 & 0,052 & 0,62 & 0,533 \\
& Homens & 200 & 4,17 & 0,780 & 0,055 & 10,41 & $<0,001$ \\
\hline
\end{tabular}




\section{Discussão}

O objetivo do presente estudo foi a criação e a validação preliminar de um instrumento de satisfação corporal situacional. Buscou-se avaliar a validade de construto da escala. O instrumento se mostrou válido, apresentando uma estrutura de quatro fatores. Análises posteriores revelaram, ainda, que os fatores variaram conforme as características demográficas da amostra, como sexo e IMC, corroborando a literatura existente na área.

Dois fatores se agruparam em razão do eixo satisfação-insatisfação. Curiosamente, o fator insatisfação incluiu itens envolvendo gordura e o fator satisfação incluiu itens sobre musculosidade. Estrutura semelhante pode ser observada na MBAS (Tylka \& cols., 2005) que possui um fator que inclui gordura e outro que inclui músculos. A Escala de Avaliação da Satisfação com a Imagem Corporal - EASIC (Ferreira \& Leite, 2002) apresentou um fator de preocupação com o peso e outro de satisfação geral. Pode-se dizer que houve uma estrutura semelhante no sentido em que itens relativos a peso e os de satisfação se agruparam em fatores distintos.

Como se pôde observar na Tabela 2 , as mulheres obtiveram uma média significativamente maior que os homens no fator "insatisfação e gordura", que possui itens de avaliação negativa do corpo, interpretados como insatisfação com o corpo todo ou com certas partes. A maioria dos itens aqui agrupados têm conteúdos relativos à gordura, como "Se estivesse mais magro(a) me sentiria muito melhor". Tal se observa com os itens de maior carga fatorial nesse fator, como o "Acho que tenho gordura demais no meu corpo", e o "Estou descontente com a minha barriga". Também entra nesse fator o item "Gostaria de mudar muitas coisas no meu corpo". Pode-se inferir que o desejo de mudança corporal agrupase com o descontentamento com a gordura e a valorização da magreza.

Itens sobre insatisfação relativa à massa muscular, como "Acho que minhas pernas são muito flácidas" e "Acho que meus braços são muito flácidos" não entraram no fator "insatisfação e gordura". Esse último não atingiu carga fatorial acima de 0,32 em nenhum fator. O primeiro entrou no fator "partes inferiores". Pode-se inferir que foi da junção entre teor negativo da questão e uma característica corporal específica, e não desses aspectos isolados, que os itens se agruparam no fator "insatisfação e gordura". A maioria dos itens desse fator tratou uma insatisfação com o corpo todo, tendo apenas as partes de cintura e barriga entrado nesse fator. A literatura tem apontado que essa região do corpo tem relevância tanto para a satisfação corporal feminina (Grogan, 2008) quanto para a masculina (Grogan, 2008; Standford \& McCabe, 2002; Tylka \& cols., 2005). Esse fator trata aspectos corporais relevantes para homens e mulheres. Ambos dão importância a essas partes do corpo na satisfação corporal e desejam ter menos gordura corporal (Grogan, 2008; Standford \& McCabe, 2002; Tylka \& cols., 2005).

Conforme se observa na Tabela 2, os homens tiveram escores maiores no fator "partes externas". Todos os seus itens traziam a satisfação com alguma parte do corpo, como pele, cabelos, pelos e rosto. Desse modo, infere-se que os homens se mostraram mais satisfeitos com essas partes do que as mulheres. Contudo, esse fator não obteve alta correlação com os demais. Isso pode ser explicado pela particularidade como foram incluídos no conjunto de questões, tendo como base a validação semântica. É importante salientar que esse não é o único instrumento a colocar esse tipo de item. O BASS também possui itens sobre cabelos e rosto (Cash, 2000). Porém, por trás da baixa relação entre esses itens e os demais pode estar a relação entre gordura e massa muscular que pareceu mais forte nos outros fatores. Esse aspecto pode ter causado maior correlação entre os três fatores e menor com esse outro. Em pesquisas futuras podem ser adicionados outros itens na escala, que não incluam o eixo gorduramúsculo, para verificar como ela se comporta.

Homens e mulheres tiveram médias similares no fator "satisfação e músculo". Os itens com maior carga fatorial foram "De modo geral, estou satisfeito(a) com o tamanho dos meus músculos", seguido pelo empate entre "Em geral, estou satisfeito(a) com a minha definição muscular" e "Estou satisfeito(a) com a grossura dos meus braços". Todos os itens são de avaliações positivas. Conforme anteriormente discutido, os que inicialmente continham avaliações negativas de musculosidade não entraram nesse fator. Nesse sentido, novamente, parece ter havido uma interação entre sentido da avaliação e conteúdo da questão na determinação do fator. Observa-se que os itens sobre músculo entraram no mesmo fator que os de satisfação geral, como o item "Estou satisfeito(a) com meu 
corpo", o que pode indicar que a amostra entendeu satisfação relacionada à massa muscular. Ainda, essa compreensão foi indistinta ao gênero. Esse resultado pode levar ao raciocínio de que tanto homens quanto mulheres entendem que satisfação com o próprio corpo inclui massa muscular, em algum nível. Esse achado é condizente com a literatura, que salienta a importância que os homens dão à musculosidade na sua imagem corporal (Grogan, 2008; Olivardia, 2002; Standford \& McCabe, 2002; Tylka \& cols., 2005). Para mulheres, a questão da musculosidade também tem chamado a atenção de pesquisadores. A última versão do instrumento de internalização de padrões socioculturais de corpo feminino, o SATAQ-3 (Thompson \& cols., 2004), inclui um fator "internalização atleta". Segundo os autores, o foco na musculosidade parece um componente de crescente importância também para as mulheres.

O fator "partes inferiores" teve um escore maior entre os homens. A média significativamente maior indica que os homens estão mais satisfeitos que as mulheres, também em relação às partes inferiores do corpo. Esse fator incluiu itens com carga fatorial negativa em relação aos demais, que foram invertidas. Desse modo, uma maior média de resposta implica maior satisfação com as partes relativas à metade inferior do corpo. O item de maior carga fatorial foi "Acho que tenho celulites em excesso", seguido de "Estou satisfeito(a) com o tamanho dos meus quadris". Sabe-se que a celulite costuma se manifestar mais nessas áreas do corpo das mulheres. Os dados corroboram a literatura da área. Ela aponta que as partes mais baixas do corpo, incluindo abdômen, coxas, quadris e glúteos, são mais relacionadas com a insatisfação corporal feminina (Grogan, 2008; Standford \& McCabe, 2002; Tylka \& cols., 2005).

$\mathrm{O}$ fato de ter havido diferenças significativas de médias em 3 dos 4 fatores sugere que o instrumento foi sensível ao gênero. Em geral, os homens tiveram pontuações maiores nos fatores que incluíam satisfação e menores no fator de insatisfação, como se pode observar na Tabela 2. Essa noção está de acordo com a literatura, que trata das intensas pressões sociais normativas de magreza feminina impactando negativamente na sua satisfação corporal (Cafri \& cols., 2005; Grogan, 2008; Jackson, 2002; Thompson \& cols., 2003). Desse modo, faz sentido que universitárias brasileiras também sofram mais pressões sociais para ter um corpo adequado aos padrões, resultando em uma menor satisfação corporal. Tal suposição não surpreende, na medida em que aparentemente são as mulheres que lideram o consumo de remédios para emagrecer. Em Minas Gerais, elas são responsáveis pela compra de $88 \%$ do total de anorexígenos vendidos (Carneiro, Guerra Junior \& Acurcio, 2008).

A relação entre a satisfação corporal e o IMC se torna evidente tendo em vista que esse teve relações mais fortes com os fatores de satisfação e insatisfação. O fator "insatisfação e gordura" foi o mais sensível ao IMC, uma vez que obteve a maior correlação. Como esta foi positiva, quanto maior o IMC maior a insatisfação. Assim, de acordo com esse fator, pessoas com maior massa corporal se mostram menos satisfeitas. A correlação negativa entre o IMC e o fator "satisfação e músculo" indica que enquanto aumenta a massa corporal, diminui a satisfação corporal relacionada à musculosidade. É compreensível que a menor das correlações envolvendo IMC seja com o fator "partes externas". Sabendo que ele inclui itens como cabelos e pelos corporais, espera-se que não haja muita variação nesses itens em função do eixo gordura-músculo, que parece balizar o entendimento da escala.

\section{Conclusões}

Uma das limitações deste estudo foi a uniformidade da amostra de estudantes universitários. Um possível caminho de pesquisas futuras seria a validação numa amostra mais representativa da população brasileira. Ela poderia ser feita com seleção randômica de participantes, para teste da estrutura fatorial. Outra possibilidade é aplicar a escala em grupos específicos, como pacientes de transtorno alimentar, pessoas que fazem dietas e/ou exercícios, dentre outros. Contudo, se o intuito for utilizar o instrumento para medir traço de satisfação corporal, seria necessário adaptá-lo. Para tal, pode-se retirar da sua instrução o pedido da autoavaliação "no presente momento", alterando-se o foco na satisfação situacional para o traço de satisfação.

Apesar da mudança conceitual no instrumento ser uma opção, grande contribuição dessa medida é levar em conta o papel do contexto na satisfação corporal. A escala pode ser aplicada em pessoas em locais específicos, como restaurantes, academias, praias, shoppings, dentre outros. Seria avaliada a possibilidade de diferenças significativas em função do ambiente social. Se o construto satisfação corporal momentânea estiver 
sendo bem avaliado, espera-se que os resultados da escala variem em situações nas quais mudanças na avaliação corporal sejam estimuladas. Nesse sentido, o instrumento trará uma contribuição interessante para aqueles que buscam entender certos aspectos do meio social e cultural que podem produzir efeitos na autoavaliação corporal dos indivíduos.

A maior contribuição desta pesquisa foi disponibilizar um instrumento válido de satisfação corporal em língua portuguesa. Os indicadores psicométricos sugerem a sua validade de construto. Observou-se, também, que alguns fatores foram mais sensíveis às mulheres do que aos homens, ou à massa corporal, indicando que o instrumento foi sensível a essas características da amostra. Seria interessante que estudos posteriores avaliassem a adequação da estrutura fatorial. Uma possível agenda de pesquisa poderia incluir estudos de revalidação da escala. Sugerem-se, ainda, estudos de validação convergente com outros instrumentos existentes sobre imagem corporal.

Retomando os dados oficiais, o relatório anual de 2007 da Junta Internacional de Fiscalização de Entorpecentes (JIFE) mostra que o Brasil assume o primeiro lugar na venda de anorexígenos, deixando para trás os Estados Unidos e a Argentina. Ademais, de acordo com a Sociedade Brasileira de Cirurgia Plástica, no ano de 2004 o Brasil realizou mais de 600 mil cirurgias plásticas. As lipoaspirações ficaram em primeiro lugar no ranking das cirurgias estéticas. Esses dados podem estar ilustrando o desejo partilhado por inúmeros brasileiros de mudança corporal de gordura e peso. Nesse contexto, o estudo da avaliação cognitiva e afetiva que um indivíduo faz do próprio corpo pode ser muito útil para compreender melhor os fatores que podem estar por trás de decisões, como o consumo de remédios que afetam o comportamento alimentar ou mesmo a busca por cirurgias estéticas.

\section{Referências}

Avalos, L., Tylka, T. L. \& Wood-Barcalow, N. (2005). The Body Appreciation Scale: Development and psychometric evaluation. Body Image, 2, 285-297.

Cafri, G., Yamamiya, Y., Brannick, M. \& Thompson, J. K. (2005). The influence of sociocultural factors on body image: A metaanalysis. Clinical Psychology: Science and Practice, 12, 421-433.
Hirata, E., Pilati R. Escala Situacional de Satisfação Corporal

Carneiro, M. F., Guerra Junior, A. A. \& Acurcio, F. A. (2008). Prescrição, dispensação e regulação do consumo de psicotrópicos anorexígenos em Belo Horizonte, Minas Gerais, Brasil. Cadernos de Saúde Pública, 24, 1763-1772.

Cash, T. F. (2000). User's manual for the Multidimensional Body-Self Relations Questionaire. Norfolk, VA: Old Dominion University.

Cash, T. F., Fleming, E. C., Alindogan, J., Steadman, L. \& Whitehead, A. (2002). Beyond body image as a trait: The development and validation of the Body Image States Scale. Eating Disorders, 10, 103-113.

Cooper, P. J., Taylor, M. J., Cooper, Z. \& Fairburn, C. G. (1987). The development and validation of the Body Shape Questionnaire. International Journal of Eating Disorders, 6, 485-494.

Costa, C., Ramos, E., Barros, H. Torres, A. R., Severo, M. \& Lopes, C. (2007). Propriedades psicométricas do Eating Disorders Inventory em adolescentes portugueses. Acta Médica Portuguesa, 20, 511-524.

Costello, A. B. \& Osborne, J. W. (2005). Best practices in exploratory factor analysis: Four recommendations for generating the most from you analysis. Practical Assessment Research \& Evaluation, 10, 1-9.

Durkin, S. J., Paxton, S. J. \& Sorbello, M, (2007). An integrative model of the impact of exposure to idealized female images on adolescent girls' body satisfaction. Journal of Applied Social Psychology, 37, 1092-1117.

Eagly, A. H. \& Chaiken, S. (1998). Attitude structure and function. Em D. T. Gilbert, S. T. Fiske \& G. Lindzey (Orgs.). The Handbook of Social Psychology (vol. 1, pp. 269-322). Nova Iorque: McGraw-Hill.

Enzmann, D. (1997). RanEigen: A program to determine the parallel analysis criterion for the number of principal components. Applied Psychological Measurement, 21, 232.

Ferreira. M. C. \& Leite, N. G. M. (2002). Adaptação e validação de um instrumento de avaliação de satisfação com a imagem corporal. Avaliação Psicológica, 2, 141-149.

Fingeret, M. C., Gleaves, D. H. \& Pearson, C. A. (2004). On the methodology of body image 
assessment: the use of figural rating scales to evaluate body dissatisfaction and the ideal body standards of women. Body Image, 1, 207212.

Freitas, S., Gorestein, C. \& Appolinário, J. C. (2002). Instrumentos para a avaliação dos transtornos alimentares. Revista Brasileira de Psiquiatria, 24, 34-38.

Garner, D. M., Olmstead, M. P., \& Polivy, J. (1983). Development and validation of a Multidimensional Eating Disorder Inventory for anorexia nervosa and bulimia. International

Journal of Eating Disorders, 2, 15-34.

Groesz, L. M., Levine, M. P. \& Murnen, S. K. (2002). The effect of experimental presentation of thin media images on body satisfaction. International Journal of Eating Disorders, 31, 1-15.

Grogan, S. (2008). Body image: Understanding body dissatisfaction in men, women, and children. East Sussex: Routledge.

Harari, D., Furst, M., Kiryati, N., Caspi, A. \& Davidson, M. (2001). A computer-based method for the assessment of body-image distortions in anorexia-nervosa patients. IEEE Transactions on Information Technology in Biomedicine, 5, 311-319.

Hart, T. A., Flora, D. B., Palyo, S. A., Fresco, D. M., Holle, C. \& Heimberg, R. G. (2008). Development and examination of the Social Appearance Anxiety Scale. Assessment, 15, 4859.

Jackson, L. (2002). Physical attractiveness: A sociocultural perspective. Em Cash, T. F. \& Pruzinsky, T. (Orgs.). Body image: A handbook of theory, research and clinical practice (pp. 13-21). Nova Iorque: The Guillford Press.

Kagawa, M., Kerr, D., Dhaliwal, S, Hills, A. P. \& Binns, C. W. (2006). Applicability of the Somatomorphic Matrix computer program in Japanese and Australian Caucasian males in relation to measured body composition. Body Image, 3, 385-394.

Kakeshita, I. S. \& Almeida S. (2006). Relação entre índice de massa corporal e a percepção da auto-imagem em universitários. Revista Saúde Pública, 40, 497-504.

Keel, P. K., Mitchell, J. E., Davis, T. L. \& Crow, S. J. (2001). Relationship between depression and body dissatisfaction between women diagnosed with bulimia nervosa. International Journal of Eating Disorders, 30, 48-56.

Kim, J-H. \& Lennon, S. L. (2007). Mass media and self-esteem, body image and eating disorder tendencies. Clothing and Textiles Research Journal, 25, 3-23.

Olivardia, R. (2002). Body image and muscularity. Em T. F. Cash \& T. Pruzinsky (Orgs.). Body image: $A$ bandbook of theory, research and clinical practice (pp. 210-218). Nova Iorque: The Guillford Press.

Pasquali, L (1999). Testes referentes a construto: teoria e modelos de construção. Em L. Pasquali (Org.). Instrumentos psicológicos: manual prático de elaboração (pp. 37-71). Brasília: LABPAM/IBAPP.

Pelletier, L. G. \& Dion, S. C. (2007). An examination of general and specific motivational mechanisms for the relation between body dissatisfaction and eating behaviors. Journal of Social and Clinical Psychology, 26, 303-333.

Pruzinsky, T. \& Cash, T. F. (2002). Understanding body images. Em T. F. Cash \& T. Pruzinsky (Orgs.). Body image: A bandbook of theory, research and clinical practice (pp. 3-12). Nova Iorque: The Guillford Press.

Scagliusi, F. B., Alvarenga, M., Polacow, V. O., Cordás, T. A. Queiroz, G. K. O., Coelho, D., Philippi, S. T. \& Lancha Jr., A H. (2006). Concurrent and discriminant validity of the Stunkard's Figure Rating Scale adapted into Portuguese. Appetite, 47, 77-82.

Sepúlveda, A. R., Botella, J. \& León, J. A. (2002). Body-image disturbance in eating disorders: A meta-analysis. Psychology in Spain, 6, 83-95.

Standford, J. N. \& McCabe, M. P. (2002). Body image ideal among males and females: Sociocultural influences and focus on different body parts. Journal of Health Psychology, 7, 675-684.

Stice, E. (1994). Review of the evidence for a sociocultural model of bulimia nervosa and an exploration of the mechanisms of action. Clinical Psychology Review, 14, 633-661.

Strahan, E. J., Wilson, A. E., Cressman, K. E. \& Buote, V. M. (2006). Comparing to perfection: How cultural norms for appearance affect 
social comparisons and self-image. Body Image, 3, 211-227.

Tabachnick, B. \& Fidell, L. (1996). Using Multivariate Statistics. ( $4^{\text {a }}$ ed.). Nova Iorque: HarperCollins.

Thompson, J. K. (2004). The (mis)measurement of body image: Ten strategies to improve assessment for applied and research purposes. Body Image, 1, 7-14.

Thompson, J. K., Van den Berg, P., Roehrig, M., Guarda, A. S. \& Heinberg, L. J. (2004). The Sociocultural Attitudes Towards

Appearance Scale-3 (SATAQ-3): Development and validation. International Journal of Eating Disorders, 53, 293-304.

Trampe, D., Siero, F. W. \& Stapel, D. A. (2007). On models and vases: Body dissatisfaction and proneness to social comparison effects. Journal of Personality and Social Psychology, 92, 106-118.

Tylka, T. L., Bergeron, D. \& Schwartz, J. P. (2005). Development and psychometric evaluation of the Male Body Attitudes Scale (MBAS). Body Image, 2, 161-175.

Van den Berg, P., Paxton, S. J., Keery, H., Wall, M. Guo, J. \& Neumark-Sztainer, D. (2007). Body dissatisfaction and body comparison with media images in males and females. Body Image, 4, 257-268.

Recebido em abril de 2009 Reformulado em dezembro de 2009 Aprovado em janeiro de 2010

Sobre os autores:

Elizabeth Hirata é graduada pela Universidade de Brasília. Terminou o mestrado em Psicologia pela mesma instituição em agosto de 2009, no Departamento de Psicologia Social e do Trabalho. Seus interesses de pesquisa são: psicologia social, imagem corporal, comportamento alimentar, cognição social, metodologia de pesquisa em psicologia social, psicologia organizacional (ensino a distância), construção de medidas psicossociais.

Ronaldo Pilati é graduado (1999) e Doutorado (2004) em Psicologia pela Universidade de Brasília, professor adjunto do Departamento de Psicologia Social e do Trabalho e do programa de pós-graduação em Psicologia Social, do Trabalho e das Organizações (PSTO) da UnB. Seus interesses de pesquisa são em psicologia social: percepção social, comportamento pró-social, cognição social, metodologia de pesquisa em psicologia social, construção de medidas psicossociais, avaliação de programas sociais e organizacionais e psicologia forense. 